\title{
Effect of Intrauterine Injection of Human Chorionic Gonadotropin Before Frozen-Thawed Embryo Transfer on Implantation and Clinical Pregnancy Rate: A Randomized Controlled Trial
}

\author{
Robab Seyed Hosseini, Laya Farzadi*, Sedigheh Abdollahi, Mohammad Nouri, Alieh Ghasemzadeh, \\ Kobra Hamdi, Hassan Soleimanpour
}

\begin{abstract}
Objectives: This study aimed to assess the effect of intrauterine injection of human chorionic gonadotropin (hCG) before frozenthawed embryo transfer on implantation and clinical pregnancy rates.

Materials and Methods: In this prospective randomized clinical trial, 100 infertile women younger than 40 years old were randomly divided into intervention $(n=50)$ and control groups $(n=50)$. Intervention group received intrauterine injection of 500 IU hCG before frozen-thawed embryo transfer, while control group had the embryo transferred without intrauterine hCG injection. Then, both groups were assessed in terms of implantation, clinical pregnancy and ongoing pregnancy rates. $\mathrm{P}<0.05$ was considered statistically significant.

Results: There was a significant difference in clinical pregnancy rate between intervention (28\%) and control (10.4\%) groups $(P=0.028$, OR: 4.28 , CI: $1.25-14.14)$. The difference in ongoing pregnancy rate between intervention $(28 \%)$ and control $(8.3 \%)$ groups was also statistically significant ( $P=0.012$, OR: 3.44 , CI: 1.1-1.18).

Conclusion: Intrauterine injection of hCG (500 IU) before frozen-thawed embryo transfer can significantly improve clinical and ongoing pregnancy in patients with history of in vitro fertilization/intracytoplasmic sperm injection (IVF-ICSI).

Keywords: Embryo transfer, Human chorionic gonadotropin, Implantation, Pregnancy
\end{abstract}

\section{Introduction}

Infertility is defined as failure to achieve pregnancy following regular intercourse for 12 months or more, without contraception; it happens in $10 \%-15 \%$ of couples (1). Despite major advances in clinical and laboratory techniques of in vitro fertilization (IVF), fertility success rate is still around $30 \%$ per cycle (2). It is estimated that more than half of lost pregnancies are due to failure of embryo implantation (1). Successful implantation, following IVF/ embryo transfer (IVF/ET) depends upon several factors, such as quality of the embryo and endometrial receptivity. One of the most important and well-known of these factors is human chorionic gonadotropin (hCG) hormone $(1,2)$.

hCG is one of the first hormones to be secreted by the embryo before implantation, and sends a message inducing growth of maternal blood vessel. Before implantation, and by binding to its endometrial receptor, hCG directly stimulates vascular growth and promotes expression of angiogenic factors such as vascular endothelial growth factor (VEGF) (1).

Ovarian stimulation usually leads to production of greater number of embryos for fresh ET (3). Through advances in embryo culture techniques, number of embryos trans- ferred in each cycle is reduced, resulting in reduced likelihood of multiple pregnancy and subsequent complications. On the other hand, occasionally we do not transfer embryo due to possible ovarian hyperstimulation syndrome or inappropriate endometrium. Hence, cryopreservation and frozen-thawed embryo replacement is an important part of assisted reproductive technology (ART) programs (3). Frozen embryo replacement cycles contribute to around $25 \%$ of all births achieved by ART (3). There is also the hypothesis that embryos may be damaged in vitrification process and secretion of hCG may be impaired. Accordingly, in this study, we tried to assess the effect of intrauterine injection of hCG 500 IU on implantation and pregnancy rate before frozen-thawed embryo transfer. Then, if the effect was positive, pregnancy rate can be increased by optimization of frozen embryo transfer (FET) cycles.

\section{Materials and Methods}

This randomized double-blind clinical trial was conducted on patients referred to Alzahra Hospital Fertility Center in Tabriz from May 2014 to April 2015.

The following patients were excluded: Patients with a history of uterine surgery such as myomectomy, patients 
with a history of recurrent miscarriage, and patients with known hydrosalpinx, endometrioma and endometriosis. Patients underwent ultrasound on 1-3 day of menstrual cycle. In the absence of ovarian cysts and intrauterine space-occupying lesions and in appropriate endometrial condition, preparation of endometrium was initiated with hormone replacement protocol, which involved administration of estrogen, followed by progesterone without ovarian downregulation (3). Administration of estradiol valerate tablets started at a dose of $4 \mathrm{mg} /$ day, which increased to $6 \mathrm{mg} /$ day after three days. Seven days later, ultrasound control was performed and estradiol dose was adjusted accordingly. When endometrial thickness reached or exceeded $8 \mathrm{~mm}$, intramuscular injection of 50 $\mathrm{mg}$ /day of progesterone hormone (Iran Hormone, Tehran, Iran) was administered. Eight-cell embryo transfer and blastocyst transfer were performed three days and five days later, respectively. During embryo transfer, patients were randomly divided (according to table of random numbers) into control and case groups (50 patients each). Case group received intrauterine injection of 40 $\mu \mathrm{l}$ of a 5000 unit hCG vial (Choriomon, IBSA, Lugano) mixed with $0.4 \mathrm{ml}$ of ISM1-type media (equivalent to 500 hCG units) through Labotect catheter (Labotect GmbH, Labor-Technik-Gottingen, Germany). Seven minutes later, embryo transfer was performed using sterile Labotect catheter, guided by abdominal ultrasound at $1-1.5 \mathrm{~cm}$ from uterine fundus. In the control group, embryo transfer was carried out with no intervention. Luteal phase was supported by injection of $100 \mathrm{mg} /$ day/IM of progesterone. Serum $\beta$ hCG test was requested two weeks after ET. With positive $\beta$ hCG, initial ultrasound was performed three weeks after embryo transfer and further ultrasounds were carried out afterwards to investigate the presence of gestational sac, position and quantity of sacs, fetal pole and heartbeat. Progesterone was continued until the 11th week of pregnancy. This study aimed to assess the effect of intrauterine hCG injection on pregnancy rate in fro- zen-thawed ET cycles. Accordingly, the following criteria were used:

- Implantation rate (IR); defined as the number of gestational sacs containing fetal pole and regular heartbeat for every transferred embryo.

- Biochemical pregnancy rate; defined as quantitative biochemical data on $\beta$ hCG in blood or urine tests, matching standard in vitro values.

- Clinical pregnancy (CP) rate (CPR); defined as confirmation of pregnancy following biochemical phase through ultrasound evidence of gestational sacs and embryo and fetal heartbeat.

- Ongoing pregnancy (OP) rate (OPR); defined as successful completion of pregnancy for 20 or more gestational weeks.

Study flowchart is shown in Figure 1. Collected data were analyzed in SPSS version 15. Descriptive results are presented as mean \pm standard deviation (SD), frequency and percentage. The relationship between qualitative variables was assessed using chi-square test (and Fisher exact test if required) and the relationship between qualitative and quantitative variables was assessed by $t$ test. Normal distribution of data was verified using Kolmogorov-Smirnov test. $P<0.05$ was considered as significant.

\section{Results}

No significant difference was found between the two groups in terms of age and quantity of transferred embryos or embryo transfer order $(P>0.05$; Table 1$)$. In the case group, $94 \%$ of embryo transfer occurred in cleavage stage and $6 \%$ in blastocyst stage, and in the control group, $90 \%$ in cleavage stage and $10 \%$ in blastocyst stage (Table 1).

In the case group, $28 \%$ had biochemical pregnancy and in the control group $20 \%$, with no significant difference between the two groups $(P>0.05$; Table 2$)$. No significant difference was found in implantation rate between case $(0.11 \pm 0.21)$ and control $(0.05 \pm 0.14)$ groups $(P>0.05$; Table 2). Multiple pregnancies were $4 \%$ in the case group

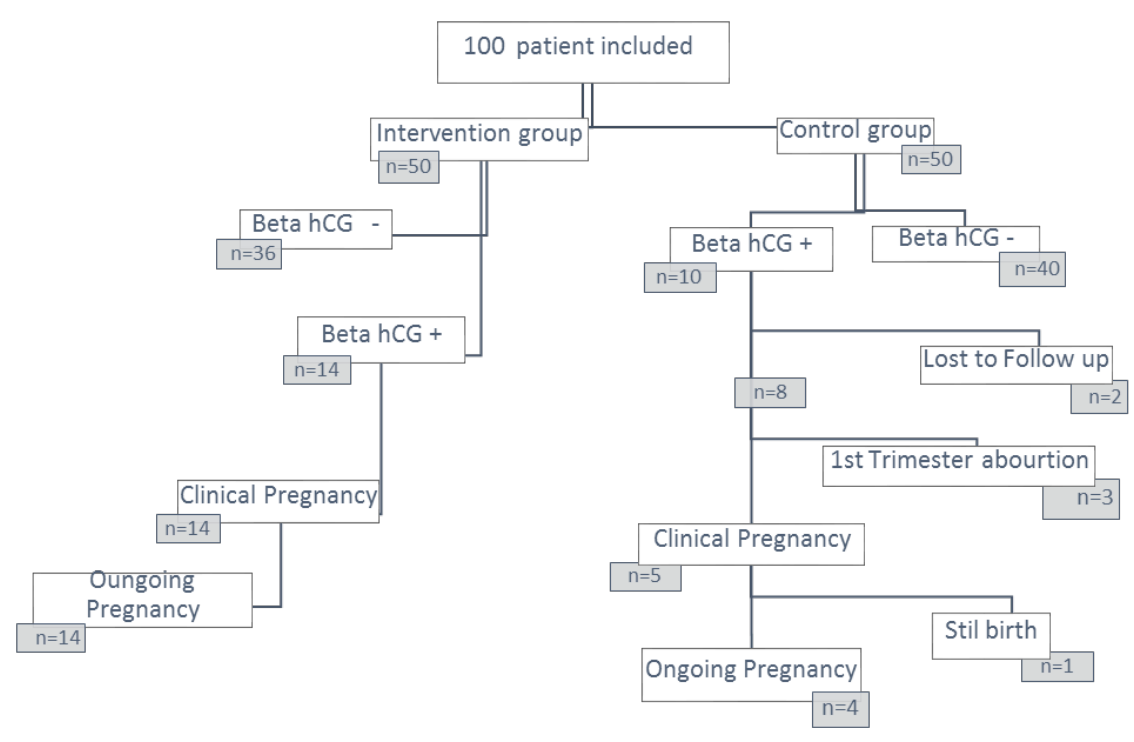

Figure 1. Flowchart of Study. 
Table 1. Demographic Characteristics of Groups

\begin{tabular}{lccc}
\hline & Case $(\mathbf{n}=\mathbf{5 0})$ & Control $(\mathbf{n}=\mathbf{5 0})$ & $\boldsymbol{P}$ \\
\hline Age (mean) & $30.5 \pm 4.85$ & $31.30 \pm 5.01$ & 0.443 \\
Embryo No/ET cycle & $2.94 \pm 068$ & $2.90 \pm 0.61$ & 0.759 \\
ET & $2.26 \pm 1.17$ & $2.32 \pm 1.12$ & 0.819 \\
Cleavage embryo & $47(94 \%)$ & $45(90 \%)$ & NA
\end{tabular}

Abbreviation: ET, embryo transfer time.

Table 2. Ongoing Pregnancy, Clinical, Biochemical and Implantation Rates in Groups

\begin{tabular}{lllc}
\hline Pregnancy Rate & $\begin{array}{l}\text { Control } \\
\text { Group }\end{array}$ & $\begin{array}{l}\text { Intervention } \\
\text { Group }\end{array}$ & $\boldsymbol{P}$ \\
\hline Ongoing pregnancy rate & $4(8.3 \%)$ & $14(28 \%)$ & 0.012 \\
No. of clinical pregnancies & $5(10.4 \%)$ & $14(28 \%)$ & 0.028 \\
No. of biochemical pregnancies & $10(20 \%)$ & $14(28 \%)$ & 0.35 \\
Implantation rate & $15.0 \pm 05.0$ & $21.0 \pm 12.0$ & 0.09 \\
\hline
\end{tabular}

and $2.1 \%$ in the control. Miscarriage in the control group was $8.3 \%$, and none in the case group. Mean number of embryos in positive and negative clinical pregnancy was $3 \pm 0.39$ and $2.9 \pm 0.76$ respectively in the case group, with no significant difference $(P=0.61)$, and $3 \pm 0$ and $2 \pm 0.61$ respectively in the control group, with no significant difference $(P=0.09)$. Mean number of embryos in positive and negative ongoing pregnancy was $3 \pm 0.96$ and $2.9 \pm 0.55$ respectively in the case group, with no significant difference $(P=0.702)$, and $3 \pm 0$ and $2.84 \pm 0.6$ respectively in the control group, with no significant difference $(P=0.6)$. No significant relationship was found between quantity of embryos and implantation in the case group with $\mathrm{r}=0.013$ and $P=0.93$, or in the control group with $\mathrm{r}=0.034$ and $P=0.82$. In case and control groups, no significant relationship was found between positive cases of clinical pregnancy, ongoing pregnancy and implantation and mean quantity of embryos $(\mathrm{P}>0.05)$. Clinical pregnancy was $28 \%$ in the case group and $10.4 \%$ in the control, with a significant difference between them $(P=0.028$, $\mathrm{OR}=4.28$, CI: 1.25-14.14), (Table 2). Ongoing pregnancy was $28 \%$ in the case group and $8.3 \%$ in the control, with significant difference between them $(P=0.012$, OR $=3.44$, CI: 1.1-1.18) (Table 2).

\section{Discussion}

This study aimed to assess the effect of intrauterine injection of 500 IU of hCG before frozen-thawed embryo transfer. Results obtained showed no significant effect on implantation or biochemical pregnancy rates, but improvements were achieved in CP and OP outcomes. The effects of hCG on implantation and pregnancy have already been studied. Several factors such as quality of embryo and endometrial receptivity are involved in implantation and the resulting successful pregnancy. The complex process of implantation is affected by many factors and one of the most important and well-known is hCG hormone (1).
One of the first studies on the effect of hCG on human endometrium was conducted by Lich et al, which showed inhibition of intrauterine Insulin like growth factor binding protein-1 (IGFBP-1) and Macrophage-Colony Stimulating Factor (M-CSF) due to intrauterine injection of 500 IU of hCG. On the other hand, increasing leukemia inhibitory factor (LIF) and VEGF (as potent pro-angiogenic factors) and matrix metalloproteinase-9 (MMP-9) leads to improved implantation conditions (4). A study by Mansour et al, conducted on patients with the first ICSI/ IVF cycle and fresh embryos showed improvement in implantation and pregnancy rates due to intrauterine injection of 500 IU of hCG before embryo transfer (2).

The present study was conducted on frozen-thawed embryos; some of patients had a history of IVF failure, no difference was observed between the control and case groups in implantation and pregnancy rates. Damage to embryos caused by potential stress of cryopreservation or changes caused by freezing process such as hardening of zona pellucida may be a reason for failure in these cases (3). On the other hand, since good quality embryos are used in the first ET cycles, embryos used in transfer cycles in the present study appear to have been of poor quality. However, as far as clinical pregnancy is concerned, the present study results concur with those found in Mansour study. Thus, in these cases, perhaps hCG improves angiogenic conditions, resulting in more stable pregnancies. In a study by Riboldi et al in Brazil, RhCG injection six hours before vitrified blastocysts transfer led to improved implantation and clinical pregnancy rates (5). In a study by Hong et al, intrauterine injection of hCG 500 IU before blastocyst ET showed no improvement in implantation and ongoing pregnancy (6). A study conducted by Wirleitner et al in 2015 showed that intrauterine HCG injection prior to ET does not improve the pregnancy and live birth rates independently of blastocyst quality. Furthermore hCG's effect on live birth rate (LBR) was similar between women over the age of 38 and under the age of 38 (7). These three studies used blastocyst. Perhaps, secretion of hCG had already started in embryos that reached high-grade blastocyst stage and injection of further hCG will have a slight effect on the results. However, embryos in cleavage stage may still benefit from intrauterine injection of hCG. A study conducted in Iran by Zarei et al showed that intrauterine injection of recombinant hCG before ET improves OPR, IR and CPR (8). The difference between this and the present study in IR was probably due to use of high quality fresh embryos in the first IVF/ET cycle. However, the two studies produced similar OPR and CPR results. Another difference between the two studies was in the use of recombinant hCG instead of urinary hCG. A study by Alvaro Santibanez et al using intrauterine injection of hCG 500 IU before embryos transfer showed an increase in IR and CPR (9). In Rebolloso et al study, injection of urinary hCG before fresh ET showed no positive effect on implantation and ongoing pregnancy results and only multiple pregnancies increased. Their study cannot be relied upon due to its small sample size (10). A systemic review 
and meta-analysis conducted by Ye et al in 2015 showed that intrauterine hCG injection prior to ET significantly increased the rates of biochemical, clinical and ongoing pregnancy compared with the control group. There were no between group difference in implantation or miscarriage ratio. This meta-analysis significantly confirms our study outcomes (11). Intrauterine hCG injection before ET is a relatively simple procedure that requires no particular training, nor is it costly and time-consuming, and hCG preparation requires no specific tools. On the other hand, dummy ET (experimental ET before actual ET) that has been accepted in many articles and references and is performed in many centers has no particular complications and compared to dummy ET even low dose of hCG, is no more invasive. Thus, in hCG injection, catheter path is recognized similar to dummy ET, which reduces ET problems. Thawed embryos are responsible for $25 \%$ of ART live births (3). Therefore, hCG injection can be performed in a relatively broad group of IVF patients with improved pregnancy outcomes.

Study limitations included use of only one center, at which, patients were referred to other physicians for pregnancy care following positive results and initial follow-ups, making careful follow-up until childbirth and assessment of LBR impossible. Moreover, since morphologically best embryos are usually transferred in the first fresh ET cycle and low-grade embryos are kept for later cycles; this may be one of the reasons for poor results obtained in the present study. Like in many other studies, in the present study, no accurate embryo grading and matching method existed. In the present study, many confounding factors were eliminated, resulting in reduced number of patients. Perhaps more tangible results could have been obtained with larger sample size.

\section{Conclusion}

Intrauterine injection of hCG before ET can increase clinical and ongoing pregnancy rates. However, further and larger studies are required before this procedure can be routinely performed. Perhaps, in future studies, assessment of live birth rate can be helpful.

\section{Ethical Issues}

Study protocol was approved by the Center for Research in Women's Health and the Ethics Committee of Tabriz University of Medical Sciences (IRCT2014110612146N5; http://en.search.irct.ir/view/20692). Explanations and written information were provided to 100 eligible patients and informed consent was obtained from them. Women selected for inclusion in study met the following criteria: (a) female under 40 years old, (b) previous IVF/ICSI history, and (c) frozen embryo grade A or B.

\section{Conflict of Interests}

The authors declare no conflict of interests.

\section{Finnacial Support}

Women's Reproductive Health Research Center, Tabriz
University of Medical Sciences, Tabriz, Iran supported this study.

\section{Acknowledgments}

The authors are grateful to all the health staff and patients who participated in the study, in addition to the staff of the infertility ward of Tabriz Alzahra hospital and Women's Reproductive Health Research center. This article is based on a dataset forming part of our proposal design, registered with the same title at women"s reproductive Health center at Tabriz University of Medical Sciences in 2014.

\section{References}

1. Fritz MA, Speroff L. Clinical Gynecologic Endocrinology and Infertility. 8th ed. USA: Lippincot Williams and Wilkins; 2011.

2. Mansour R, Tawab N, Kamal O, et al. Intrauterine injection of human chorionic gonadotropin before embryo transfer significantly improves the implantation and pregnancy rates in in vitro fertilization/ intracytoplasmic sperm injection: a prospective randomized study. Fertil Steri 2011;96(6):1370-4.e1. doi: 10.1016/j.fertnstert.2011.09.044.

3. Gardner DK, Weissman A, Howles CH, Shoham Z. Textbook of Assisted Reproductive Techniques: Laboratory and Clinical Perspective. United Kingdom: Martin Dunitz Ltd; 2012.

4. Licht P, Losch A, Dittrich R, Neuwinger J, Siebzehnrubl E, Wildt L. Novel insights into human endometrial paracrinology and embryo-maternal communication by intrauterine microdialysis. Hum Reprod Update. 1998;4(5):532-8.

5. Riboldi M, Barros B, Piccolomini M, Alegretti JR, Motta ELA, Serafini PC. Does the intrauterine administration of rhCG before vitrified blastocysts transfer improves the potential of pregnancies when using blastocysts of inferior morphological grading? Ferti Steril. 2013;100(3):S289. doi: 10.1016/j. fertnstert.2013.07.1080.

6. Hong KH, Forman EJ, Werner MD, et al. Endometrial infusion of human chorionic gonadotropin at the time of blastocyst embryo transfer does not impact clinical outcomes: a randomized, double-blind, placebocontrolled trial. Fertil Steril. 2014;102(6):1591-5.e2. doi: 10.1016/j.fertnstert.2014.08.006.

7. Wirleitner B, Schuff M, Vanderzwalmen $P$, et al. Intrauterine administration of human chorionic gonadotropin does not improve pregnancy and life birth rates independently of blastocyst quality: a randomised prospective study. Reprod Biol Endocrinol. 2015;13:70. doi: 10.1186/s12958-0150069-1.

8. Zarei A, Parsanezhad ME, Younesi M, et al. Intrauterine administration of recombinant human chorionic gonadotropin before embryo transfer on outcome of in vitro fertilization/intracytoplasmic sperm injection: A randomized clinical trial. Iranian 
J Reprod Med. 2014;12(1):1-6.

9. Santibanez A, Garcia J, Pashkova O, et al. Effect of intrauterine injection of human chorionic gonadotropin before embryo transfer on clinical pregnancy rates from in vitro fertilisation cycles: a prospective study. Reprod Biol Endocrinol. 2014;12:9. doi: 10.1186/1477-7827-12-9.

10. Rebolloso MM, Rosales De Leon JC, Galache Vega P, Santos-Haliscak R, Diaz-Spindola P, Gonzalez Vega O. Do intrauterine injection of human chorionic gonadotropin (hCG) before embryo transfer increases implantation and pregnancy rates in patients undergoing in vitro fertilization? Fertil Steril. 2013;100(3):S289. doi:10.1016/j. fertnstert.2013.07.1082.

11. Ye H, Hu J, He W, Zhang Y, Li C. The efficacy of intrauterine injection of human chorionic gonadotropin before embryo transfer in assisted reproductive cycles: Meta-analysis. J Int Med Res. 2015;43(6):738-46. doi: 10.1177/0300060515592903.

Copyright $\odot 2016$ The Author(s); This is an open-access article distributed under the terms of the Creative Commons Attribution License (http://creativecommons.org/licenses/by/4.0), which permits unrestricted use, distribution, and reproduction in any medium, provided the original work is properly cited. 